Pacific Journal of Mathematics

SOLVABLE AND SUPERSOLVABLE GROUPS IN WHICH
EVERY ELEMENT IS CONJUGATE TO ITS INVERSE 


\section{SOLVABLE AND SUPERSOLVABLE GROUPS IN WHICH EVERY ELEMENT IS CONJUGATE TO ITS INVERSE}

\section{J. L. BERGGREN}

Let $\subseteq$ be the class of finite groups in which every element is conjugate to its inverse. In the first section of this paper we investigate solvable groups in $\varsigma_{\text {: }}$ in particular we show that if $G \in \subseteq$ and $G$ is solvable then the Carter subgroup of $G$ is a Sylow 2-subgroup and we show that any finite solvable group may be embedded in a solvable group in $\subseteq$. In the second section the main theorem reduces the study of supersolvable groups in $\sqrt{S}$ to the study of groups in $\sqrt{S}$ whose orders have the form $2^{\alpha} p^{\beta}, p$ an odd prime.

Notation. The notation here will be as in [1] with the addition of the notation $G=X Y$ to mean $G$ is a split extension of $Y$ by $X$. Also, $F(G)$ will denote the Fitting subgroup of $G$ and $\Phi(G)$ the Frattini subgroup of $G$. We will denote the maximal normal subgroup of $G$ of odd order by $O_{2^{\prime}}(G)$. Further, $\operatorname{Hol}(G)$ will denote the split extension of $G$ by its automorphism group.

If $K$ and $T$ are subgroups of $G$ we will call $K$ a $T$-group if $T \leqq N_{G}(K)$ and we say $K$ is a $T$-indecomposable $T$-group if $K=$ $K_{1} \times K_{2}$, where $K_{1}$ and $K_{2}$ are $T$-groups, implies $K_{1}=\langle 1\rangle$ or $K_{2}=\langle 1\rangle$.

1. Burnside [2] proved that if $P$ is a Sylow $p$-subgroup of the finite group $G$ and if $X$ and $Y$ are $P$-invariant subsets of $P$ which are not conjugate in $N_{G}(P)$ then they are not conjugate in $G$. Using Burnside's method one may prove a similar fact about the Carter subgroups. The proof is easy and we omit it.

Lemma 1.1. Let $C$ be a Carter subgroup of the solvable group $G$ and let $A$ and $B$ be subsets of $C$, both normal in $C$. If $A \neq B$ then $A$ and $B$ are not conjugate in $G$.

Theorem 1.1. If $G$ is a solvable group in $\subseteq$ then a Carter subgroup of $G$ is a Sylow 2-subgroup of $G$.

Proof. Let $C$ be a Carter subgroup of $G$. If $C$ has a nonidentity element of odd order then $C$ has a nonidentity central element $g$ of odd order, since $C$ is nilpotent. Then with $A=\{g\}$ and $B=\left\{g^{-1}\right\}$ the hypotheses of Lemma 1.1 are satisfied and, since $A \neq B, g$ and $g^{-1}$ are not conjugate in $G$, contradicting our supposition that $G \in \widetilde{S}$. 
Hence $C$ is a 2-group. As $C$ is self-normalizing in $G, C$ must be a Sylow 2-subgroup of $G$.

Note. This proof implies, also, that $Z(C)$ is an elementary abelian 2-group. However, the theorem of Burnside we mentioned can be used to show that if $T$ is a Sylow 2-subgroup of any group $G \in \mathbb{S}$ (whether solvable or not) then $Z(T)$ is elementary abelian. Thus, if $G \in \mathfrak{S}$ and $T$ is a Sylow 2-subgroup of $G$ the ascending central series of $T$ has elementary abelian factors.

CoRollary 1.1. If $T$ is a Sylow 2-subgroup of a solvable group $G \in \mathfrak{S}$ then $N_{G}(T)=T$.

Proof. By Theorem $1.1 T$ is a Carter subgroup of $G$. Carter subgroups are self-normalizing.

COROLlary 1.2. If $G$ and $T$ are as in Corollary 1.1, and if $T$ is abelian, then $G$ has a normal 2-complement.

Proof. By Corollary 1.1 and the assumption $T$ is abelian, $T$ is in the center of its normalizer. The result follows from a well-known theorem of Burnside.

We now investigate two families of solvable groups in $\mathfrak{\subseteq}$.

TheOREm 1.2. If $G \in \mathbb{S}$ and a Sylow 2-subgroup of $G$ is cyclic then $G=T K$ where $K$ is an abelian normal subgroup of odd order and $T=\langle\alpha\rangle$ with $\alpha^{2}=1$ and $g^{\alpha}=g^{-1}$ for all $g \in K$.

Proof. As $G$ has a cyclic Sylow 2-subgroup, $G$ is solvable. By Corollary $1.2 G=T K, T=\langle\alpha\rangle$ is a Sylow 2-subgroup of $G$ and $K$ is a normal subgroup of odd order. By the Note after Theorem 1.1, $\alpha^{2}=1$. If $\alpha$ did not induce a fixed-point-free automorphism of $K$ then $C_{G}(T) \cap K \supsetneq\langle 1\rangle$, so $N_{G}(T) \supsetneq T$, contradicting Corollary 1.1. Thus $g \rightarrow g^{\alpha}$ is a fixed-point-free automorphism of $K$. It is known that if $K$ has a fixed-point-free automorphism $\alpha$ of order 2 then $\alpha(k)=k^{-1}$ for all $k \in K$ and hence $K$ is abelian.

TheOREm 1.3. Let $G$ be a finite solvable group in 5 and suppose a Sylow 2-subgroup $T$ of $G$ has order 4 . Then $T$ is elementary abelian, $G$ has a normal 2-complement $K$, and $K^{(1)}$ is nilpotent.

Proof. As $G$ is solvable, Corollary 1.1 and 1.2 imply that $G=$ 
$T K$ where $|T|=4$ and $K$ is a normal subgroup of odd order. The Note after Theorem 1.1 implies $T$ is elementary, say $T=\langle\alpha\rangle \times\langle\beta\rangle$. Let $K_{\alpha}$ and $K_{\beta}$ denote the set of fixed points of the automorphisms of $K$ induced by $\alpha$ and $\beta$ respectively. Then $\langle 1\rangle=C_{K}(T) \supseteqq K_{\alpha} \cap K_{\beta}$. Hence, as $T$ is abelian, $K_{\alpha}$ is $\beta$-invariant and $\beta$ induces a fixed-point free automorphism of $K_{\alpha}$. Thus $K_{\alpha}$ is abelian. Then, by [4], $K^{(1)}$ is nilpotent.

Finally, we show that any finite solvable group can be embedded in a solvable group in $\mathfrak{S}$. We shall need the following lemma.

Lemma 1.2. Let $G \in \mathfrak{S}$ and let $\langle x\rangle$ be a cyclic group of order $p$, where $p$ is an odd prime. Let $\alpha$ be an involution and define $H=$ $\langle G w\langle x\rangle, \alpha\rangle$, where $x^{\alpha}=x^{-1}$ and $b^{\alpha}=b$ for all $b \in G$. Then $H \in \mathfrak{S}$.

Proof. Let $K=G \times G^{x} \times \cdots \times G^{x p-1}$ be the base subgroup of $G w\langle x\rangle$. Then $K \in \mathfrak{S}$ since $G \in \mathfrak{S}$. Suppose $h_{1} \in H$ and

$$
h_{1}=x^{r} g_{0} \cdot g_{1}^{x} \cdots g_{p-1}^{x^{p-1}},
$$

where $r \not \equiv 0(p)$. Writing [j] for $x^{j}$ we may write

$$
h_{1}=x^{r} \cdot g_{0} \cdot g_{r}^{[r]} \cdots g_{(p-1) r}^{[(p-1) r]} .
$$

Now, if $g \in G$ then $\left(g^{[i]}\right)^{x^{r}}=g^{[i+r]}$ implies that

$$
\left(g^{[i]}\right)^{-1} x^{-r} g^{[i]} x^{r}=\left(g^{[i]}\right)^{-1} g^{[i+r]},
$$

and hence $\left(g^{[i]}\right)^{-1} x^{r} g^{[i]}=x^{r}\left(g^{[i+r]}\right)^{-1} g^{[i]}$. Thus if $\beta=g_{e r}^{[(e-1) r]}$ then $\left(x^{r}\right)^{\beta}=$ $x^{r}\left(g_{e r}^{-1}\right)^{[e r]}\left(g_{e r}\right)^{[(e-1) r]}$. Writing $h_{1}^{\beta}=x^{r} \cdot f_{0} \cdot f_{r}^{[r]} \cdots f_{(p-1) r}^{[(p-1) r]}$, where $f_{i} \in G$ for all $i$, we see that $f_{i r}=g_{i r}$ if $i \neq e, e-1$ while $f_{e r}=1$. Thus first changing the rightmost $g_{i r}^{[i r]}$ in $h_{1}$ to 1 by conjugation and proceeding to the left we may conjugate $h_{1}$ to an element $h=x^{r} g$, where $g \in G=G^{[0]}$.

Pick $a \in G$ such that $g^{a}=g^{-1}$ and let $u=a a^{x} \cdots a^{x^{p-1}}$. Then with $\gamma=\alpha u x^{-r}$ we have $h^{\gamma}=h^{-1}$. It remains to consider elements of $H$ of the form $h=\alpha \cdot x^{r} \cdot g_{0} \cdot g_{1}^{[1]} \cdots g_{p-1}^{[p-1]}$, where [j] denotes $x^{j}$. If $r \not \equiv 0(p)$ then let $e$ be an integer such that $2 e \equiv-r(p)$. Then $h$ conjugated by $x^{e}$ has the form $\alpha y_{0} y_{1}^{[1]} \cdots y_{p-1}^{[p-1]}$ where the $y_{i} \in G$.

We now exploit the fact that, since $x^{\alpha}=x^{-1}$ and $g^{\alpha}=g$ for all $g \in G=G^{[0]}, g_{p-1}^{[p-1]}=\left(g_{p-1}^{[1]}\right)^{\alpha}, g_{p-2}^{[p-2]}=\left(g_{p-2}^{[2]}\right)^{\alpha}$, etc. Thus

$$
\alpha^{\Upsilon(p-1)}=\alpha\left(g_{p-1}^{-1}\right)^{[p-1]}\left(g_{p-1}\right)^{[1]},
$$

where $\gamma(p-1)=g_{p-1}^{[1]}$. Performing this computation for

$$
\gamma(p-1), \gamma(p-2), \cdots, \gamma((p+1) / 2),
$$

where $\gamma(e)=g_{e}^{[p-e]}$ and observing that $u=\gamma(p-1) \cdots \gamma((p+1) / 2)$ 
has the identity in $G^{[i]}$ as its $i$-th component for $i>((p+1) / 2)$ we see that $h^{u}$ has the form $h_{1}=\alpha \cdot f_{0} \cdot f_{1}^{[1]} \cdots f_{r}^{[r]}$ where $r=(p-1) / 2$ and $f_{i} \in G$ for all $i$. Then $h_{1}^{-1}=\alpha \cdot f_{0}^{-1} \cdot\left(\left(f_{1}^{-1}\right)^{[1]} \cdots\left(f_{r}^{-1}\right)^{[r]}\right)^{\alpha}$. Now for all $i=0, \cdots, r$ pick $a_{i} \in G$ such that $f_{i}^{a_{i}}=f_{i}^{-1}$ and let $u=a_{0} \cdot v \cdot v^{\alpha}$ where $v=a_{1}^{[1]} \cdots a_{r}^{[r]}$. Taking $x=u \alpha$ it is easy to see that $h_{1}^{x}=h_{1}^{-1}$, using the fact that $\left(v v^{\alpha}, \alpha\right)=\left(g_{0}, v v^{\alpha}\right)=1$. This disposes of all cases.

Theorem 1.4. If $G$ is a finite solvable group then there exists a solvable group $L \in \mathfrak{S}$ and a monomorphism $\tau: G \rightarrow L$.

Proof. If $G$ is abelian let $L=\langle G, \alpha\rangle$ where $\alpha^{2}=1$ and $g^{\alpha}=g^{-1}$ for all $g \in G$. Then in $L$ every element of $G$ is conjugate to its inverse and all other elements lie in the coset $G \alpha$ which consists of involutions, so $L \in \subseteq$ and $L$ is solvable. Hence the theorem is true for all abelian groups $G$. Induct on $|G|$ and assume it is true for all solvable groups of order less than the order of $G$. Now let $H \triangleleft G$ such that $[G: H]=p, p$ a prime. Our induction hypothesis says there is a solvable $K \in \mathbb{S}$ and a monomorphism of $H w C_{p}$ into $K w C_{p}$, where $C_{p}$ is cyclic of order $p$. By Satz 15.9 [3] (Chapter I) there is a monomorphism of $G$ into $H w C_{p}$, so $G$ may be imbedded in $K w C_{p}$. If $p=$ 2 then by Theorem 1.1 of [1] $K w C_{p} \in \mathfrak{S}$, and it is solvable since $K$ is. If $p>2$ then by Lemma $1.2 K w C_{p}$ has a solvable extension $\left\langle K w C_{p}, \alpha\right\rangle \in \mathfrak{S}$.

Thus, in this case as well, $G$ may be imbedded in a solvable group in $\mathfrak{s}$.

This concludes our investigation of solvable groups in $\mathfrak{\subseteq}$.

2. In $\S 1$ we showed that if $G \in \subseteq$ is a solvable group with an abelian Sylow 2-subgroup $T$ then $T$ has a normal complement in $G$. Of course, if $G$ is supersolvable then (by the Sylow Tower Theorem) $T$ has a normal complement $K$, regardless whether $T$ is abelian or $G \in \mathfrak{S}$. If we assume that $G \in \mathfrak{S}$, where $G$ is supersolvable, then with the above notation we assert.

THEOREM 2.1. The Sylow 2-subgroup $T$ is in $\mathfrak{S}$, and $K$ and $\Phi(T)$ are contained in $F(G)$.

Proof. That $T \in \subseteq$ was remarked in [1]. Since $G$ is supersolvable $G^{(1)} \leqq F(G)$. Now $G \in \subseteq$ implies $G / G^{(1)} \in \subseteq$ and since $G / G^{(1)}$ is abelian $G / G^{(1)}$ is an elementary abelian 2-group. Thus $\Phi(T) \leqq G^{(1)}$, and since $(2,|K|)=1, K \leqq G^{(1)}$.

REMARK. If $G \in \mathcal{S}$ is supersolvable Theorem 2.1 implies $G$ is a 
split extension of a nilpotent group $K$ by a two-group $T$ in $\mathfrak{S}$. If $S$ is a Sylow 2-subgroup of $F(G)$ then $S \triangleleft G$, so $G / S \in \mathcal{S}$. But by Theorem 2.1 G/S is isomorphic to a split extension $E K$ of the nilpotent group $K$ by an elementary abelian two-group $E$. Thus given a supersolvable $G$ in $\subseteq$ there exists a supersolvable $G^{*} \in \mathfrak{S}$ such that $O_{2^{\prime}},\left(G^{*}\right) \cong O_{2^{\prime}},(G)$ but $G^{*}$ has an elementary abelian Sylow 2-subgroup. Now let $G=T K \in \subseteq$ be given, where $G$ is supersolvable and $T$ and $K$ are as above. Let $P_{1}, \cdots, P_{r}$ be the Sylow subgroups of $K$, so $K=P_{1} \times \cdots \times P_{r}$. If $\pi_{i}$ is the projection of $K$ onto $P_{i}$ let $H_{i}=$ $\operatorname{ker}\left(\pi_{i}\right)$. Then $H_{i} \triangleright G$ and $G / H_{i} \cong T P_{i}$, a split extension of $P_{i}$ by $T$ which is supersolvable and in $\subseteq$. We have now reduced the study of supersolvable groups in $\subseteq$ to two questions:

(1) Given a 2-group $T \in \subseteq$ S and a $p$-group $P$ ( $p$ an odd prime) find the split extensions $T P$ of $P$ by $T$ which are supersolvable and in $\mathfrak{S}$.

(2) Given split extensions $T P_{1}, \cdots, T P_{n}$ of $P_{i}$-groups by $T$ (where

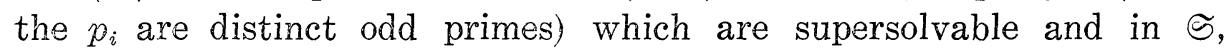
when is $T P_{1} \wedge T P_{2} \wedge \cdots \wedge T P_{n} \in \subseteq$ ? (For a definition of the symbol $\wedge$ see [3], Satz 9.11.)

The answer to (2) is not "Always." For example let

$$
T P_{1}=\langle x, y, a, b\rangle
$$

where $\langle x, y\rangle$ is the non-abelian group of order 27 and exponent 3, $\langle a, b\rangle$ is the four-group, and $(x, a)=x,(x, b)=1,(y, a)=1,(y, b)=y$. Let $T P_{2}=\langle u, v, a, b\rangle$ where $\langle u, v\rangle$ is the nonabelian group of order 125 and exponent 5 with $(u, a)=u,(u, b)=1,(v, a)=1,(v, b)=v$. Then $T P_{1}$ and $T P_{2}$ are supersolvable and in $\varsigma^{5}$, but $T P_{1} \wedge T P_{2} \notin \subseteq$.

The next theorem answers (1) when $T$ and $P$ are abelian. It may be used to show that for certain $P$ no $T$ exists such that $T P \in \mathbb{S}$.

THEOREM 2.2. If $G=T K$ is a group in $\subseteq$ such that $K$ is abelian of odd order $(K \triangleleft G)$ and $T$ is an abelian two-group then $T$ is elementary and we may pick a basis $x_{1}, \cdots, x_{n}$ for $K$ and a basis $\alpha, \beta_{1}, \cdots, \beta_{m}$ for $T$ such that $x_{i}^{\gamma}=x_{i}^{-1}$ for all $i=1, \cdots, n$ and $x_{i}^{\beta}{ }^{j}=x_{i}^{ \pm 1}$ for all $i, j$. Conversely any such group is in $\mathfrak{S}$.

Proof. Since $G / K \cong T, T \in \subseteq$ S. Being abelian $T$ must be elementary. Since $K$ is a finite $T$-group we may write $K=K_{1} \times \cdots \times K_{n}$ where each $K_{i}$ is a $T$-indecomposable $T$-group. Now pick any $\gamma \in T$. Since $|\gamma| \leqq 2$ and $K_{i}$ is abelian of odd order, $K_{i}=I_{\gamma} \times F_{\gamma}$ where

$$
I_{\gamma}=\left\{x \in K_{i} \mid x^{\gamma}=x^{-1}\right\} \text { and } F_{\gamma}=\left\{x \in K_{i} \mid x^{\gamma}=x\right\} .
$$


(For clearly $K_{i} \geqq I_{\gamma} \times F_{\gamma}$. For any $x \in K_{i}$ let $z=x x^{\gamma}$ and $w=x\left(x^{-1}\right)^{r}$. Observe that $z \in F_{r}, w \in I_{r}$, and $x^{2}=z w$. Since $x^{2} \in I \times F_{\gamma}$ and $K_{i}$ has odd order, $x \in I_{\gamma} \times F_{\gamma}$. Thus $K_{i}=I_{\gamma} \times F_{\gamma}$.) Since $T$ is abelian and $K_{i}$ is a $T$-group, $I_{\gamma}$ and $F_{\gamma}$ are also $T$-groups. But $K_{i}$ is $T$-indecomposable so $I_{\gamma}=\langle 1\rangle$ or $F_{\gamma}=\langle 1\rangle$. This means that each $\gamma \in T$ either inverts every element of $K_{i}$ or fixes every element of $K_{i}$. Hence in any decomposition of $K_{i}$ as a direct product of cyclic groups each direct factor is a $T$-group. As $K_{i}$ is $T$-indecomposable we conclude $K_{i}$ is cyclic. Let $K_{i}=\left\langle x_{i}\right\rangle$. Because $G \in \mathfrak{S}$ there exists $\alpha \in T$ such that $\left(x_{1} \cdots x_{n}\right)^{\alpha}=x_{1}^{-1} \cdots x_{n}^{-1}$. Hence $x_{i}^{\alpha}=x_{i}^{-1}$ for all $i$ and therefore $x^{\alpha}=x^{-1}$ for all $x \in K$. Now let $\alpha, \beta_{1}, \cdots, \beta_{m}$ be a basis of $T$, where $\alpha$ is as above. We found that for an arbitrary $\gamma \in T$ and an arbitrary $x \in K_{i}, x^{\gamma}=x$ or $x^{\gamma}=x^{-1}$. Hence for each $j$ and $i, x_{i}^{\beta} j=x_{i}$, where $\varepsilon= \pm 1$.

Conversely, if $G=T K$ is as in the conclusion of the theorem then $g \in G$ either has the form $x_{1}^{e_{1}} \cdots x_{n}^{e_{n}}$ (which is conjugated to its inverse by $\alpha$ ) or the form $\gamma x_{1}^{e_{1}} \cdots x_{n}^{e_{n}}$, with $\gamma \in T$. In this case it is easy to see that $g^{\beta}=g^{-1}$, where $\beta=\gamma \alpha$.

As an example of how this theorem might be applied we shall show that if $P=\left\langle x, y \mid x^{p^{n-1}}=y^{p}=1, x^{y}=x^{1+p^{n-2}}\right\rangle$, where $p$ is an odd prime and $n \geqq 3$, then there is no two-group $T$ and supersolvable extension $T P$ such that $T P \in \mathcal{S}$. For suppose there were such a $T$, with $T P \in \mathcal{S}$. We may assume, by previous remarks, that $T$ is elementary abelian. Then $T P / \Phi(P) \in \mathcal{S}$ and by the foregoing theorem there exists $\alpha \in T$ such that $x^{\alpha}=x^{-1} x^{p k}$ and $y^{\alpha}=y^{-1} x^{p e}$. Then

$$
\left(x^{y}\right)^{\alpha}=\left(x^{1+p^{n-2}}\right)^{\alpha}=x^{-1-p^{n-2}} x^{p k}
$$

while $\left(x^{\alpha}\right)^{y^{\alpha}}=\left(x^{-1} x^{p k}\right)^{y^{-1}}=\left(x^{-1}\right)^{y^{-1}} x^{p k}=x^{-1+p^{n-2}} x^{p k}$. Since $\left(x^{y}\right)^{\alpha}=\left(x^{\alpha}\right)^{y^{\alpha}}$ we conclude that $x^{-p^{n-2}}=x^{p^{n-2}}$. Therefore $x^{2 p^{n-2}}=1$, contradicting the supposition that $p$ is odd. Hence no such $G$ exists.

3. We now give an example of a solvable group satisfying the hypotheses of Theorem 1.3 which does not have a nilpotent normal 2-complement. Thus the second assertion of Theorem 2.1 does not generalize to solvable groups with a normal 2-complement. Let

$$
H=\left\langle x, y, z \mid x^{7}=y^{3}=z^{2}=1, x^{y}=x^{2}, x^{z}=x^{-1}, y^{z}=y\right\rangle,
$$

so $H=\mathrm{Hol}\left(C_{7}\right)$, where $C_{7}$ is a cyclic group of order 7 . Let

$$
C_{2}=\left\langle u \mid u^{2}=1\right\rangle
$$

and define $K=H w C_{2}$. In $K$ let $a=x, b=x^{u}, c=y\left(y^{2}\right)^{u}, d=z z^{u}, e=$ $u$, and consider the subgroup $G=\langle a, b, c, d, e\rangle$. Then $G$ has defining 
relations $a^{7}=b^{7}=c^{3}=d^{2}=e^{2}=1,(a, b)=(c, d)=(d, e)=1, a^{d}=a^{-1}$, $b^{d}=b^{-1}, a^{c}=a^{2}, b^{c}=b^{4}, c^{e}=c^{-1}$, and $a^{e}=b$.

Consider the subgroup $\langle a, b, d, e\rangle$. Elements of the form $e a^{i} b^{j}$, $a^{i} b^{j}, d a^{i} b^{j}$, and $e d a^{i} b^{j}$ are conjugated to their inverses by, respectively, $a^{j} d e a^{-j}, d, 1$ and $e$. We may now consider elements $c^{\varepsilon} e^{i} d^{j} a^{k} b^{m}, \varepsilon= \pm 1$. Such an element is always conjugate to an element of the form! $c e^{i} d^{j} a^{k} b^{m}$. Now $c e d a^{k} b^{m}$ and $c e a^{k} b^{m}$ are conjugated to their inverses by $c e$ and ced respectively. Finally $c a^{k} b^{m}$ and $c d a^{k} b^{m}$ are conjugated to their inverses by $a^{k} b^{5 m} e a^{-k} b^{-5 m}$ and $a^{2 k} b^{4 m} e a^{-2 k} b^{-4 m}$ respectively.

This completes the proof that $G \in \mathfrak{S}$. Notice $G$ satisfies the hypotheses of Theorem 1.3 but the normal 2-complement $K=\langle a, b, c\rangle$ is not nilpotent. In fact $F(K)=K^{(1)}$.

\section{REFERENCES}

1. J. L. Berggren, Finite groups in which every element is conjugate to its inverse, Pacific J. Math., 28 (1969), 289-293.

2. W. Burnside, Theory of Groups of Finite Order, Dover, New York, 1955.

3. B. Huppert, Endliche Gruppen I, Springer-Verlag, New York, 1967.

4. J. N. Ward, Involutory automorphisms of groups of odd order, J. Australian Math. Soc., 6 (1966), 480-494.

Received February 10, 1970.

SIMON FraSer UNIVERSITY 



\section{PACIFIC JOURNAL OF MATHEMATICS}

\section{EDITORS}

H. SAMELSON

Stanford University

Stanford, California 94305

C. R. Новву

University of Washington

Seattle, Washington 98105
J. DUGundJI

Department of Mathematics

University of Southern California

Los Angeles, California 90007

RICHARD ARENS

University of California

Los Angeles, California 90024

\section{ASSOCIATE EDITORS}
E. F. BECKENBACH
B. H. NeumanN
F. WOLF
K. YOSHIDA

\section{SUPPORTING INSTITUTIONS}
UNIVERSITY OF BRITISH COLUMBIA
CALIFORNIA INSTITUTE OF TECHNOLOGY
UNIVERSITY OF CALIFORNIA
MONTANA STATE UNIVERSITY
UNIVERSITY OF NEVADA
NEW MEXICO STATE UNIVERSITY
OREGON STATE UNIVERSITY
UNIVERSITY OF OREGON
OSAKA UNIVERSITY
UNIVERSITY OF SOUTHERN CALIFORNIA
STANFORD UNIVERSITY
UNIVERSITY OF TOKYO
UNIVERSITY OF UTAH
WASHINGTON STATE UNIVERSITY
UNIVERSITY OF WASHINGTON
AMERICAN MATHEMATICAL SOCIETY CHEVRON RESEARCH CORPORATION TRW SYSTEMS
NAVAL WEAPONS CENTER 


\section{Pacific Journal of Mathematics}

\section{Vol. 37, No. $1 \quad$ January, 1971}

Gregory Frank Bachelis and Haskell Paul Rosenthal, On unconditionally

converging series and biorthogonal systems in a Banach space .........

Richard William Beals, On spectral theory and scattering for elliptic

operators with singular potentials .........................

J. Lennart (John) Berggren, Solvable and supersolvable groups in which every element is conjugate to its inverse ........................ 21

Lindsay Nathan Childs, On covering spaces and Galois extensions ..........

William Jay Davis, David William Dean and Ivan Singer, Multipliers and

unconditional convergence of biorthogonal expansions..............

Leroy John Derr, Triangular matrices with the isoclinal property ............

Paul Erdős, Robert James McEliece and Herbert Taylor, Ramsey bounds for

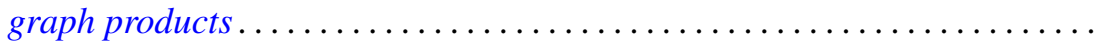

Edward Graham Evans, Jr., On epimorphisms to finitely generated

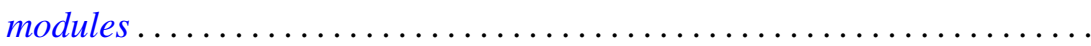

Hector O. Fattorini, The abstract Goursat problem ................. 51

Robert Dutton Fray and David Paul Roselle, Weighted lattice paths .........

Thomas L. Goulding and Augusto H. Ortiz, Structure of semiprime $(p, q)$

radicals ...........................................

E. W. Johnson and J. P. Lediaev, Structure of Noether lattices with join-principal maximal elements ....

David Samuel Kinderlehrer, The regularity of minimal surfaces defined over

slit domains

Alistair H. Lachlan, The transcendental rank of a theory. .

Frank David Lesley, Differentiability of minimal surfaces at the boundary ...

Wolfgang Liebert, Characterization of the endomorphism rings of divisible torsion modules and reduced complete torsion-free modules over complete discrete valuation rings....

Lawrence Carlton Moore, Strictly increasing Riesz norms.

Raymond Moos Redheffer, An inequality for the Hilbert transform ...

James Ted Rogers Jr., Mapping solenoids onto strongly self-entwined,

circle-like continua..........................

Sherman K. Stein, B-sets and planar maps ................... 217

Darrell R. Turnidge, Torsion theories and rings of quotients of Morita

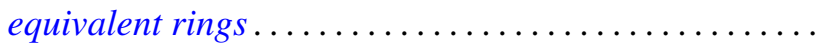

Fred Ustina, The Hausdorff means of double Fourier series and the principle of localization ................................

Stanley Joseph Wertheimer, Quasi-compactness and decompositions for arbitrary relations.

Howard Henry Wicke and John Mays Worrell Jr., On the open continuous images of paracompact $\check{C}$ ech complete spaces... 